\title{
Final Fates of Massive Stars
}

\author{
Ken'ichi Nomoto \\ Kavli Institute for the Physics and Mathematics of the Universe, University of Tokyo, \\ 5-1-5 Kashiwanoha, Kashiwa, Chiba 277-8583, Japan \\ email: nomoto@astron.s.u-tokyo.ac.jp
}

\begin{abstract}
Massive stars are thought to play important roles in the early evolution of the Universe. In this paper, we first classify the final fates of massive stars into 7 cases according to their mass ranges. These variations of the final fate may correspond to the observed large diversities of supernova properties, such as extremely faint and extremely luminous (superluminous) supernovae, and the extremely energetic hypernovae. We then focus on the properties of the peculiar superluminous Type Ic supernova 1999as. We examine radioactive decay models, magnetar models, and circumstellar interaction models for the light curve of SN 1999as. We find that these models are not quite successful, and thus it is crucially important to improve these models to clarify the final fates of massive stars.
\end{abstract}

Keywords. supernova, hypernova, superluminous, nucleosynthesis

\section{Final Fates of Massive Stars}

The final stages of massive star evolution, supernova properties, and their chemical yields depend on the progenitor's main-sequence masses $M$ (e.g., Arnett 1996, Filippenko 1997, Nomoto et al. 2006, Smartt 2009). Here we call some specific supernovae (SNe) as follows. In terms of the kinetic explosion energy $E$, we use "Hypernovae" for such energetic SNe as $E_{51}=E / 10^{51} \mathrm{erg}>10$. In terms of brightness, we use "Faint SNe" for low luminosity SNe, and "Superluminous SNe (SLSNe)" for SNe brighter than -21 mag at maximum (Quimby in this volume).

The following mass ranges are set by various types of criteria, based on some combinations of observations and models. But the criteria and critical masses are not quite systematic yet, and should still be regarded as working hypothesis (Nomoto et al. 2009, 2010).

(1) $M_{\text {up }}-10 M_{\odot}$ stars: Faint supernovae: These stars become electron capture SNe because their degenerate $\mathrm{O}+\mathrm{Ne}+\mathrm{Mg}$ cores collapse due to electron capture. $M_{\mathrm{up}} \sim$ $9 \pm 0.5 M_{\odot}$ depending on the mass loss rate on the super-AGB phase thus on the metallicity (e.g., Pumo et al. 2009).

(2) 10 - $13 M_{\odot}$ stars: Faint Supernovae: These stars undergo Fe-core collapse to form a neutron star (NS) after the phase of strong Neon shell-flashes (Nomoto \& Hashimoto 1988). Their Fe core is relatively small, and the resultant $\mathrm{SNe}$ tend to be faint (Smartt 2009).

(3) $13 M_{\odot}-M_{\mathrm{BN}}$ stars: Normal Supernovae: These stars undergo Fe-core collapse to form a NS, and produce significant amount of heavy elements from $\alpha$-elements and Fe-peak elements. The boundary mass between the NS and black hole (BH) formation, $M_{\mathrm{BN}} \sim 25 M_{\odot}$, is only tentative.

(4) $M_{\mathrm{BN}}-\mathbf{8 0} M_{\odot}$ stars: Hypernovae and Faint Supernovae: These stars undergo Fe-core collapse to form a BH. SNe seem to bifurcate into two branches, Hypernovae and Faint SNe. If the BH has little angular momentum, little mass ejection would take 


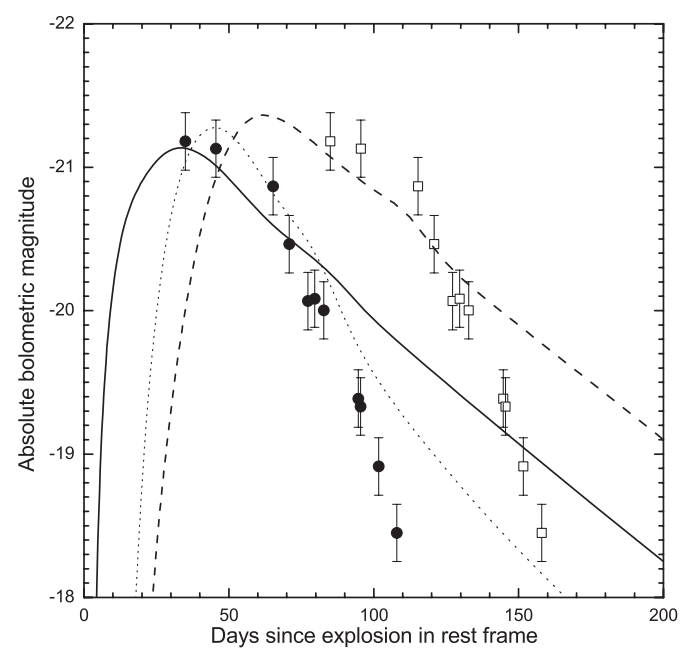

Figure 1. Model LCs with $30 M_{\odot}$ ejecta and a kinetic energy of $3 \times 10^{52} \mathrm{erg}$, compared with the observed bolometric LC of SN 1999as, with the first data epoch of $t_{0}=35$ days (filled circles) and of $t_{0}=85$ days (open squares) are shown (Deng et al. 2009). The model LC to approximately fit the former was synthesized assuming $\sim 7 M_{\odot}{ }^{56} \mathrm{Ni}$ (solid line), while the latter $\sim 9.5 M_{\odot}$ (dashed line).

place and be observed as Faint SNe. On the other hand, a rotating BH could eject a matter in a form of jets to make a Hypernova. The latter explosions produce a large amount of heavy elements from $\alpha$-elements and Fe-peak elements. Nucleosynthesis in these jet-induced explosions is in good agreement with the abundance patterns observed in extremely metal-poor stars.

(5) 80 - $140 M_{\odot}$ stars: Superluminous SNe (SLSNe): These massive stars undergo nuclear instabilities and associated pulsations ( $\epsilon$-mechanism) at various nuclear burning stages depending on the mass loss and thus metallicity. Eventually, these stars undergo Fe-core collapse. Depending on the angular momentum, Hypernova-like energetic SNe could occur to produce large amount ${ }^{56} \mathrm{Ni}$. (Because of the large ejecta mass, the expansion velocities may not be high enough to form a broad line features.)

Thanks to the large $E$ and ${ }^{56} \mathrm{Ni}$ mass, SNe in this mass range could be SLSNe. The upper limit of ${ }^{56} \mathrm{Ni}$ mass has been estimated to be $\sim 10 M_{\odot}$. The possible presence of circumstellar matter (CSM) leads to an energetic SN IIn. Pulsation could also cause superluminous events.

(6) 140 - $300 M_{\odot}$ stars: SLSNe: If these very massive stars (VMS) do not lose much mass, they become pair-instability supernovae (PISN). The star is completely disrupted without forming a $\mathrm{BH}$ and thus ejects a large amount of heavy elements, especially ${ }^{56} \mathrm{Ni}$ of mass up to $\sim 40 M_{\odot}$. Radioactive decays could produce SLSNe (Gal-Yam in this volume).

(7) Stars with $M \gtrsim 300 M_{\odot}$ : SLSNe: These VMSs are too massive to be disrupted by PISN but undergo core collapse (CVMS), forming intermediate-mass black holes (IMBHs). Some mass ejection could be possible, associated with the possible jet-induced explosion, which could form a superluminous SNe (SLSNe).

Here we focus on the light curve models for superluminous Type Ic supernova 1999as (Deng et al. 2009, Nugent et al. 2009) to understand the explosion mechanisms of very massive stars. We find that the light curve models for SN 1999as are not quite successful and need further improvement. 


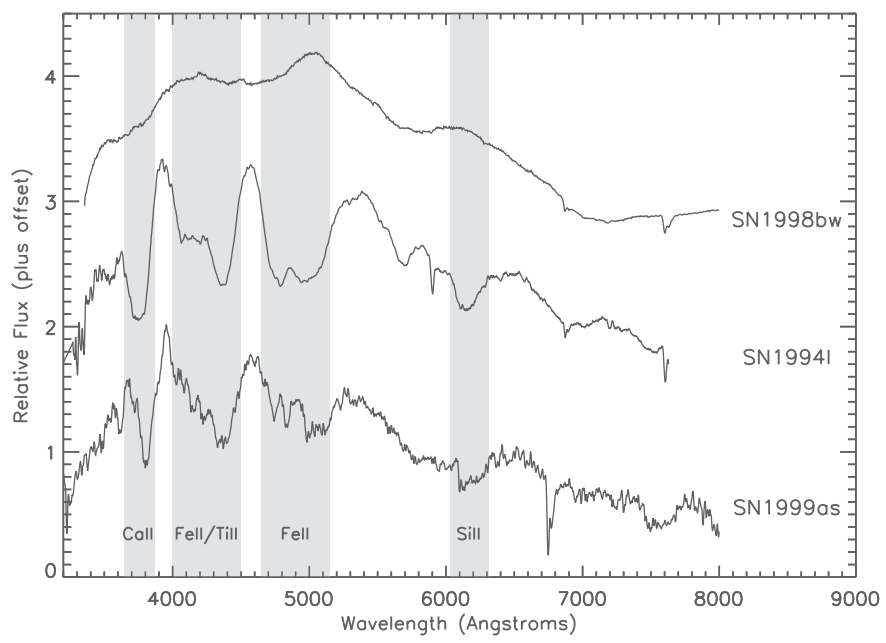

Figure 2. The spectrum of SN 1999as 25 days after discovery compared to SN 1998bw and SN 1994I (Kasen 2004).

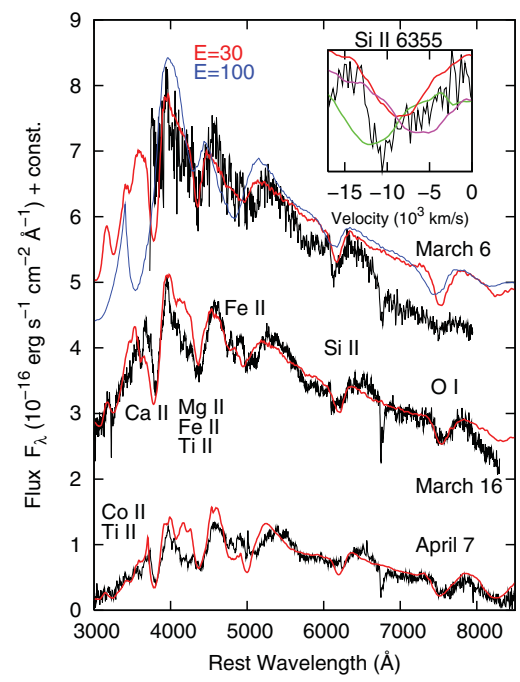

Figure 3. Synthetic spectra compared with the observed spectra taken on 1999 March 6, 16 and April 7 (from top to bottom). The red lines show synthetic spectra for the model with 30 $M_{\odot}$ ejecta and a kinetic energy of $3 \times 10^{52} \mathrm{ergs}$ (Deng et al. 2009).

\section{Superluminous Type Ic Supernova 1999as}

Supernova (SN) 1999as, discovered in a galaxy of $z=0.127$ (Knop et al. 1999), had long been the most luminous SN in terms of absolute brightness (Richardson et al. 2002, Hatano et al. 2001). With a peak magnitude of $M_{\mathrm{V}} \sim-21.5$ (Nugent et al. 2009; $m-M=38.8$ ), it is $\sim 8$ times brighter than GRB-supernova 1998bw, $\sim 3$ times brighter than Type Ia/IIn (IIa) SN 2002ic (e.g., Deng et al. 2004), and $\sim 6$ times brighter than normal Type Ia SNe. On the other hand, SN 1999as shows overall spectral similarities to normal Type Ic SN (SN Ic) 1994I (Fig. 2). 


\section{Radioactive Decay Models}

The optical LC of SN 1999as (Fig. 1), like other Type Ib/Ic SNe, was possibly powered by $\gamma$-rays and positrons released in the radioactive decays of ${ }^{56} \mathrm{Ni}$, i.e., ${ }^{56} \mathrm{Ni} \rightarrow{ }^{56} \mathrm{Co} \rightarrow{ }^{56} \mathrm{Fe}$.

\subsection{Mass of Radioactive ${ }^{56} \mathrm{Ni}$}

SN 1999as had a peak $M_{\text {bol }} \sim-21.2$ (Nugent et al. 2009), while the spectral modeling can constrain its peak epoch to be $\sim 35-85$ days after explosion. In comparison, GRB-SN 1998bw had a peak $M_{\mathrm{bol}} \sim-18.7$ and $t \sim 15$ days at light maximum, and its ${ }^{56} \mathrm{Ni}$ mass has been modeled to be $\sim 0.4 \mathrm{M}_{\odot}$ (Nakamura et al. 2001$)$. Then one gets $M\left({ }^{56} \mathrm{Ni}\right) \sim 5-9$ $\mathrm{M}_{\odot}$ for SN 1999as.

From the viewpoint of explosive nucleosynthesis, such a large amount of fresh ${ }^{56} \mathrm{Ni}$ clearly indicates that the SN explosion was extremely energetic. In a core-collapse SN, most ${ }^{56} \mathrm{Ni}$ are the outcome of complete Si-burning in the region where temperatures can reach $\gtrsim 5 \times 10^{9} \mathrm{~K}$ (i.e., $T_{\mathrm{Si}}$ ) as the $\mathrm{SN}$ shock wave propagates through it (Arnett 1996). The region behind the shock front is radiation dominated and has roughly the same temperature (e.g., Shigeyama et al. 1988). Thus, complete Si-burning is confined below a radius $R_{\mathrm{Ni}}$ in the pre-explosion star for a given explosion energy, $E$, and $E \sim$ $(4 \pi / 3) R_{\mathrm{Ni}}^{3} a T_{\mathrm{Si}}^{4}$, or $R_{\mathrm{Ni}} \sim 3700\left(E / 10^{51} \mathrm{ergs}\right)^{1 / 3} \mathrm{~km}$.

As the model for SN 1999as, the explosion of the $\mathrm{C}+\mathrm{O}$ core of the $M_{\mathrm{MS}}=80 \mathrm{M}_{\odot}$ star leads to a final ejecta mass of $\sim 30 \mathrm{M}_{\odot}$. As the energy rises from $1 \times 10^{52}$ to $8 \times 10^{52}$ ergs, $M\left({ }^{56} \mathrm{Ni}\right)$ increases from $\sim 1$ to $\sim 3 \mathrm{M}_{\odot}$ for $M_{\mathrm{MS}}=50 \mathrm{M}_{\odot}$, and from $\sim 1.5$ to $\sim 7$ $\mathrm{M}_{\odot}$ for $M_{\mathrm{MS}}=80 \mathrm{M}_{\odot}$.

For $M_{\mathrm{MS}}=100 \mathrm{M}_{\odot}$ star, Type Ic SN ejecta mass is $\sim 40 \mathrm{M}_{\odot}$. This model synthesize $\sim 7 \mathrm{M}_{\odot}{ }^{56} \mathrm{Ni}$ for only $E \sim 4 \times 10^{52} \mathrm{ergs}$. The synthetic spectra will be similar for the similar $E / M_{\mathrm{ej}}$.

\subsection{Synthetic Spectra of SN 1999as}

The spectra of SN 1999as, which were taken on 1999 March 6, 16 and April 17 (Nugent et al. 2009), are calculated for the $\sim 30 \mathrm{M}_{\odot}$ ejecta produced from the explosion of the $\mathrm{C}+\mathrm{O}$ core of the $80 \mathrm{M}_{\odot}$ star with a kinetic energy of $3 \times 10^{52}$ ergs.

The synthesized spectra are shown in Figure 3, and compared with the observed SN spectra. The overall fitness is satisfactory. All the absorption features are identified, being attributed to the P-Cygni lines of O I, Mg II, Si II, Ca II, Ti II, and Fe II.

The $\mathrm{Si}$ abundance is higher than the solar value, while Fe and Co are $\sim 10$ times more abundant than the solar values. This is evidence of explosive-synthesis products being mixed out into the ejecta above $8,000 \mathrm{~km} \mathrm{~s}^{-1}$.

The supernova explosion date is also a key parameter which was estimated through spectrum modeling. The observed bolometric luminosity can be taken as approximately proportional to $\left(v_{\mathrm{ph}} t\right)^{2} T_{\mathrm{eff}}^{4}$, where $t$ is the rest-frame epoch with respect to explosion and $T_{\text {eff }}$ the effective temperature. $v_{\text {ph }}$ can be measured by fitting the absorption shapes of the observed P-Cygni lines (Jeffery \& Branch 1990), so $t$ is derived. A rest-frame epoch is estimated as $t_{1} \sim 50-100$ days after explosion for the March 6 spectrum. The best-fitting model spectra discussed above correspond to $t_{1}=80$ days.

\subsection{Light Curve Models}

Both the LC assuming $t_{0}=35$ days after explosion and that of $t_{0}=85$ days are shown in Figure 1. The relative flatness of LC during the first month suggests that the SN was possibly discovered not far from its actual light maximum.

The bolometric LCs of $30 \mathrm{M}_{\odot}$ ejecta with $3 \times 10^{52}$ ergs are shown in Figure 1 . To fit the slowest LC case, i.e., $t_{0}=85$ days, a total amount of $\sim 9.5 \mathrm{M}_{\odot}{ }^{56} \mathrm{Ni}$ is required, which 


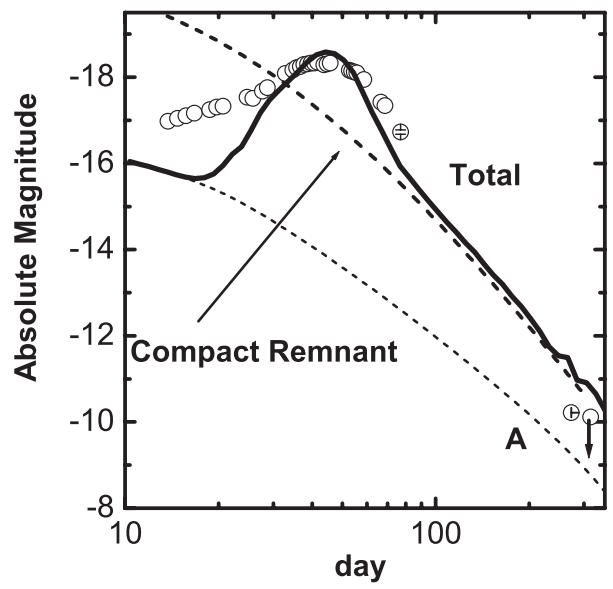

Figure 4. Synthetic light curve with the putative energy input from the central remnant (thick solid) (Maeda et al. 2007). The input luminosity of the remnant (thick dashed) and the contribution from the blob (thin dashed) are also shown.

were assumed by us to fill the inner part of the ejecta up to $\sim 7000 \mathrm{~km} \mathrm{~s}^{-1}$. The LC (dashed line) peaks $\sim 10$ days before $t_{0}$, attributable to the existence of ${ }^{56} \mathrm{Ni}$ at relatively high velocities.

\subsection{Fast Decline of the Light Curve}

The above radioactive decay models fail to reproduce the fast LC drop after $\sim t_{0}+60$ days. The observed luminosity decayed at a rate of $\gtrsim 0.06 \mathrm{mag} / \mathrm{day}$, fast as compared with other Type Ic SNe during similar epochs $(\lesssim 0.02 \mathrm{mag} /$ day; Tomita et al. 2006). In the CSM-interacting Type Ib SN 2006jc, dust formation shifted the bulk of the bolometric luminosity to the infrared (Tominaga et al. 2008, Nozawa et al. 2008). However, a pronounced red continuum should have been seen, which characterized the optical spectra of SN 2006jc at corresponding epochs (Smith, Foley, \& Filippenko 2008).

\section{Magnetar Models}

We note that a fast late LC drop has also been observed in the peculiar Type Ib SN 2005bf, which shows two peaks in the optical light curve (Fig. 4 and references therein). The first peak luminosity is reproduced if $\sim 0.07 M_{\odot}$ of ${ }^{56} \mathrm{Ni}$ is mixed out to outer layers (as a blob), and the second peak (at $\sim 40$ days) can be reproduced by the radioactive decay of $\sim 0.32 M_{\odot}{ }^{56} \mathrm{Ni}$ (Tominaga et al. 2005).

At $\sim 270$ days since the explosion, however, the absolute R-band magnitude of SN 2005bf is $\sim-10.2$ (Maeda et al. 2007). It is very faint as compared to other $\mathrm{SNe} \mathrm{Ib/c,at}$ least by 2 magnitudes (e.g., by 3 magnitudes fainter than SN 1998bw at a similar epoch). If the $\mathrm{R}$ magnitude is close the bolometric magnitude, ${ }^{56} \mathrm{Ni}$ required to fit the luminosity is only $\sim 0.03-0.08 M_{\odot}$. Why this is much smaller than $\sim 0.32 M_{\odot}$ that reproducing the early phase peak luminosity is a serious question.

Encountered by this difficulty in the ${ }^{56} \mathrm{Ni}$ heating model, Maeda et al. (2007) have adopted an alternative scenario in which the heating source is a newly born, strongly magnetized neutron star (a magnetar). A synthetic light curve is shown in Figure 4 (Maeda et al. 2007). The input luminosity of the remnant (thick dashed) and the contribution from the ${ }^{56} \mathrm{Ni}$ blob (thin dashed) for the 1st peak are shown. 


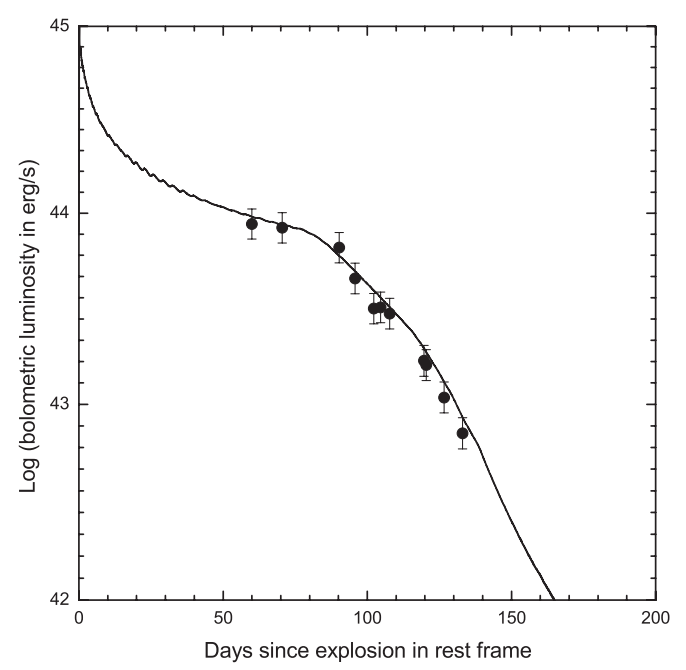

Figure 5. The LC of a CSM-interaction model (solid line) compared with the observed LC (filled circles; assuming a first data epoch of $t_{0}=60$ days). The model ejecta have $\sim 10 M_{\odot}$ and a kinetic energy of $1 \times 10^{52} \mathrm{erg}$, while the model CSM have a power-law density profile index of $n=2$ for the inner $\sim 0.90 M_{\odot}$ and of $n=5$ for the outer $\sim 0.25 M_{\odot}$ (Deng et al. 2009).

The large second peak luminosity and the rapid decline after the second peak can qualitatively be explained. The model parameters are: $L_{0}=8 \times 10^{43} \mathrm{erg} \mathrm{s}^{-1}, t_{0}=60^{\mathrm{d}}$, $\beta=4$, which corresponds to a pulsar with $B_{\mathrm{mag}} \sim 3 \times 10^{14}\left(P_{0} / 10 \mathrm{~ms}\right)^{2} \sqrt{0.1 / f_{\mathrm{rad}}}$ gauss, using the dipole radiation formula. Here $P_{0}$ is the initial spin period, $t_{0}$ is the characteristic time scale of the high energy input, and $f_{\mathrm{rad}}$ is a fraction of energy going into the radiation. A relatively large breaking index $\beta=4$ is required to reproduce the large contrast between the peak and the tail. The total energy injection with these parameters is $\sim 7 \times 10^{50}\left(f_{\mathrm{rad}} / 0.1\right)^{-1} \mathrm{erg}$, a fraction of which might be consumed to increase the kinetic energy of the SN ejecta to $E_{51}=1-1.5$ and/or to develop the pulsar nebula in the early phase. For the LC of SN 1999as, it would be interesting to apply the magnetar model (also Kasen \& Bildsten 2010, Woosley 2010).

\section{Circumstellar Interaction Model}

As seen in Figure 2, narrow spectral features are observed around 4200 and $4800 \AA$ in SN 1999as (line width $\sim 1000 \mathrm{~km} \mathrm{~s}^{-1}$ ). These features seem to be high-velocity Fe lines with $v \sim 12,000 \mathrm{~km} \mathrm{~s}^{-1}$. We note that a jet-like explosion may result in a high-velocity shell of explosive-synthesized Fe-group elements along the jet directions (Khokhlov et al. 1999, Maeda et al. 2002). Multi-dimensional radiative transfer calculations (e.g., Höflich et al. 1996, Tanaka et al. 2007) are required to investigate such scenarios.

Kasen (2004) modeled high-velocity narrow Fe II features by assuming the existence of a thin, detached shell, and suggested circumstellar interactions for the LC of SN 1999as. Deng et al. (2009) calculated the circumstellar interaction model for the LC of SN 1999as in the same way as done by Suzuki \& Nomoto (1995). The total ejecta mass is $\sim 10 \mathrm{M}_{\odot}$. The collision starts at a distance of $r_{0}=2 \times 10^{14} \mathrm{~cm}$, where the CSM density is $\rho_{0}$, and the CSM has a power-law density profile $\rho=\rho_{0}\left(r / r_{0}\right)^{-n}$.

High CSM densities and a large explosion energy are required to reproduce the brightness of SN 1999as. The density in the shocked ejecta and CSM is so high that the reverse and forward shocks are radiative to form a dense cooling shell. The regions heated by 
the forward and reverse shocks emit X-rays, a large part of which is converted into UV and optical light.

The best-fitting model LC is shown in Figure 5 (dotted line), where the observed LC is drawn adopting an epoch of 60 days for the first data point. The model explosion energy is $\sim 1 \times 10^{52}$ ergs. The CSM parameters are $\rho_{0} \sim 3.5 \times 10^{-13} \mathrm{~g} \mathrm{~cm}^{-3}, n=2$ for the inner $0.90 M_{\odot}$, and $n=5$ for the outer $0.25 M_{\odot}$. The steep outer density profile is required to reproduce the late fast LC drop. The total interacting-CSM mass is then $\sim 1.15 M_{\odot}$. The corresponding mass-loss rate would be unusually large, e.g., $\sim 0.03 M_{\odot} \mathrm{yr}^{-1}$ within $\sim 30$ years prior to the SN explosion if adopting a wind velocity of $\sim 100 \mathrm{~km} \mathrm{~s}^{-1}$.

Further refinement is necessary by considering that the CSM formed with such an extensive mass loss could be optically thick. The optical thickness would improve the early part of the model LC.

\section{Discussion}

The optical spectra and light curve of the superluminous Type Ic SN 1999as are modeled. First we apply the radioactive model, which requires $M_{\mathrm{ej}} \sim 30 \mathrm{M}_{\odot}\left(M_{\mathrm{MS}}=80 \mathrm{M}_{\odot}\right)$ and $E \sim 3 \times 10^{52}$ ergs. The high explosion energy is consistent with $\sim 6 \mathrm{M}_{\odot}{ }^{56} \mathrm{Ni}$ that are required to power the exceptional brightness of the SN. These model values of $M_{\mathrm{ej}}$ and $E$ are larger than the GRB-supernovae, i.e., SN $1998 \mathrm{bw}\left(\sim 10 \mathrm{M}_{\odot}\right.$ and $\sim 5 \times 10^{52}$ ergs; Nakamura et al. 2001), SN 2003dh $\left(\sim 7 \mathrm{M}_{\odot}\right.$ and $\sim 3.5 \times 10^{52}$ ergs; Deng et al. $2005)$, and SN 2003lw ( $\sim 13 \mathrm{M}_{\odot}$ and $\sim 6 \times 10^{52}$ ergs; Mazzali et al. 2006$)$, although the energy value is more or less comparable.

It has been demonstrated that the core-collapse explosions of massive metal-poor stars (Umeda \& Nomoto 2008) can synthesize the required large amount of ${ }^{56} \mathrm{Ni}$ of several $\mathrm{M}_{\odot}$. Nugent et al. (2009) did find that the host galaxy of SN 1999as has a relatively low metallicity.

Pair-instability SNe from $M_{\mathrm{MS}} \sim 140-270 \mathrm{M}_{\odot}$ (Barkat, Rakavy, \& Sack 1967, Bond, Arnett, \& Carr 1984, Umeda \& Nomoto 2002, Heger \& Woosley 2002) have very large $M_{\mathrm{ej}} / E$, which results in very late LC peak epochs, very slow brightness decline after the peak, and relatively narrow-lined spectra.

However, the above radioactive decay models fail to reproduce the fast decline of the light curve of SN 1999as. We thus need to explore possible alternative models.

The magnetar model has parameters to fit to the observed light curve. However, the progenitor's main-sequence mass should be smaller than $\sim 25 \mathrm{M}_{\odot}$ to produce a magnetic neutron star rather than a black hole.

The strong circumstellar interaction models for SN 1999as would require the existence of an optically-thick, non-Hydrogen shell. It's configuration would be non-spherical to avoid the contamination of the Type Ic spectral features which dominated the observed spectra. However, the presence of non-Hydrogen CSM is not trivial. The collision of massive stars (in particular, Wolf-Rayet stars) in a dense stellar cluster can make a massive $\mathrm{C}+\mathrm{O}$ star surrounded by a massive non-Hydrogen CSM (Suzuki et al. 2007).

The above models are not quite successful in explaining the properties of SN 1999as, in particular, the late fast LC drop and some narrow spectral features. It is crucially important to improve these models to clarify the final fates of massive stars.

I would like to thank Peter Nugent, Jinsong Deng, and many collaborators for the work on SN 1999as. This research has been supported in part by World Premier International Research Center Initiative, MEXT, and by the Grant-in-Aid for Scientific Research of the JSPS (23540262) and MEXT (22012003, 23105705), Japan. 


\section{References}

Aldering, G., et al. 2006, ApJ, 650, 510

Arnett, W. D. 1996, Nucleosynthesis and Supernovae (Princeton: Princeton Univ. Press)

Barkat, Z., Rakavy, G., \& Sack, N. 1967, Phys. Rev. Letters, 18, 379

Bond, J. R., Arnett, W. D., \& Carr, B. J. 1984, ApJ, 280, 825

Deng, J., et al. 2002, ApJ, 605, L37

Deng, J., Tominaga, N., Mazzali, P. A., Maeda, K., \& Nomoto, K. 2005, ApJ, 624, 898

Deng, J., et al. 2009, in preparation

Filippenko, A. V. 1997, ARAA, 35, 30

Hatano, K., Maeda, K., Deng, J., Nomoto, K., et al. 2001, in ASPC Conf. Proc. 251., New Century of X-ray Astronomy, ed. H. Inoue \& H. Kunieda (San Francisco: ASPC), 244

Herger, A. \& Woosley, S. E. 2002, ApJ, 567, 532

Höflich, P., Wheeler, J. C., Hines, D. C., \& Trammell, S. R. 1996, ApJ, 459, 307

Jeffery, D. J. \& Branch, D. 1990, in Jerusalem Winter School for Theor. Phy. V. 6, Supernovae, ed. J. C. Wheeler, T. Piran, \& S. Weinberg (Singapore: World Scientific), 149

Kasen, D. 2004, Ph.D. Thesis, Univ. California at Berkeley

Kasen, D. \& Bildsten, L. 2010, ApJ, 717, 245

Khokhlov, A. M., Höflich, P. A., Oran, E. S., et al. 1999, ApJ, 524, L107

Knop, R., et al. 1999, IAUC, 7128

Maeda. K., Nakumura, T., Nomoto, K., et al. 2002, ApJ, 565, 405

Maeda, K. et al. 2007, ApJ, 666, 1069

Mazzali, P. A., et al. 2006, ApJ, 645, 1323

Nakamura, T, Mazzali, P. A., Nomoto, K., \& Iwamoto, K. 2001, ApJ, 550, 991

Nomoto, K. \& Hashimoto, M 1988, Phys. Rep., 163, 13

Nomoto, K., et al. 2006, Nuclear Phys A 777, 424 (astro-ph/0605725)

Nomoto, K., et al. 2009, in IAU Symp. 254, The Galaxy Disk in Cosmological Context, ed. J. Andersen, et al. (Cambridge: Cambridge Univ. Press), 355 (arXiv: 0901.4536)

Nomoto, K., et al. 2010, in IAU Symp. 265, Chemical Abundances in the Universe: Connecting First Stars to Planet, ed. K. Cunha, et al. (Cambridge: Cambridge Univ. Press), 34

Nozawa, T., et al. 2008, ApJ, 684, 1343

Nugent, P., et al. 2009, private communication

Pumo, M. L., et al. 2009, ApJ, 705, L138

Quimby, R. M., Aldering, C., Wheeler, J. C., et al. 2007, ApJ, 668, L99

Richardson, D., Branch, D., Casebeer, D., et al. 2002, ApJ, 123, 745

Shigeyama, T., Nomoto, K., \& Hashimoto, M. 1988, A\&A, 196, 141

Smartt, S. J. 2009, ARAA, 47, 63

Smith, N., Foley, R. J., \& Filippenko, A. V., 2008, ApJ, 680, 568

Suzuki, T. \& Nomoto, K. 1995, ApJ, 455, 658

Suzuki, T. K., Nakasato, N., Baumgardt, H., et al. 2007, ApJ, 668, L19

Tanaka, M., Maeda, K., Mazzali, P. A., \& Nomoto, K. 2007, ApJ, 668, L19

Tomita, H., et al. 2006, ApJ, 644, 400

Tominaga, N., et al. 2005, ApJ, 633, L97

Tominaga, N., et al. 2008, ApJ, 687, 1208

Umeda, H. \& Nomoto, K. 2002, ApJ, 565, 385

Umeda, H. \& Nomoto, K. 2008, ApJ, 673, 1014

Woosley, S. E., 2010, ApJ, 719, L204 